\title{
Luminescent properties of active optical fibers
}

\author{
Jacek Żmojda ${ }^{1}$, Piotr Miluski ${ }^{1}$, Marcin Kochanowicz ${ }^{l}$, Jan Dorosz ${ }^{1}$, Agata Baranowska ${ }^{2}$ Magdalena Leśniak $^{3}$ \\ and Dominik Dorosz ${ }^{3 *}$ \\ ${ }^{1}$ Faculty of Electrical Engineering, Bialystok University of Technology, Wiejska 45A, 15-351 Bialystok, \\ ${ }^{2}$ Faculty of Mechanical Engineering, Bialystok University of Technology, Wiejska 45A, 15-351 Bialystok, \\ ${ }^{3}$ Faculty of Materials Science and Ceramics, AGH - University of Science and Technology,
} 30 Mickiewicza Av., 30-059 Krakow

Received June 17, 2019; accepted June 28, 2019; published June 30, 2019

\begin{abstract}
Luminescent optical fibres are one of the most important photonics elements as they allow to construct high power fibre lasers and different unique optical sources in the broad range from UV to IR. The most important requirements cover efficient luminescence core materials and easily pumped optical fibre constructions. Depending on applications, a variety of optical fibres have been proposed based on glasses and polymers. In the present paper some recent constructions developed by the Bialystok Photonics Group have been shown.
\end{abstract}

The development of optical lasers, amplifiers, and broadband sources of amplified spontaneous emission is closely connected with research on new constructions of optical fibers doped with luminescent centres like rare earth (RE) ions and organic dyes. In our research, we frequently showed that a full knowledge about spectroscopic properties of active materials as a perspective medium in optical fiber is essential to analyse the impact of both structural and spectroscopic parameters. They are determined by the type of core glass i.e. (level of phonon energy vibrations), concentration and ratio of luminescence dopants used and, in result, define laser parameters, technology of optical fiber drawing process, as well as fiber geometry: diameter and position of the core, shape of the inner clad, power of pumping radiation and the length of optical fiber on its luminescence spectrum. In addition, optimization of such parameters as: the type and concentration of an active dopant, absorption and emission - cross-sections, lifetimes, efficiency of energy transfer between lanthanides and thermal parameters of core glass enable to obtain luminescence in a visible and near-infrared range [1].

In glass optical fibre manufacturing, the selection of matrix is crucial. Among oxide glasses, special attention has been recently paid to antimony and germanium-based matrices, due to their combination of chemical durability, low phonon energies ( 600 to $\left.800 \mathrm{~cm}^{-1}\right)$ and high transparency of a wide range.

*ddorosz@agh.edu.pl
The main advantage of antimony glasses is the ability to create a wide glassy area among such glass-forming oxides like $\mathrm{GeO}_{2}, \quad \mathrm{SiO}_{2}, \quad \mathrm{P}_{2} \mathrm{O}_{5}, \quad \mathrm{~B}_{2} \mathrm{O}$, which, in consequence, increase their thermal stability required in optical fiber production. Another interesting feature is the role of $\mathrm{Sb}_{2} \mathrm{O}_{3}$ as a mild reducing agent for noble metal ions. This mild reduction property enables in-situ reduction of $\mathrm{Ag}^{+}$to $\mathrm{Ag}^{0}$ in a one-step process of melting, which provides a simple, low-cost method of preparing bulk plasmonic materials. Germanate glasses also represent an interesting group of modern matrix which is characterized by favourable physicochemical and optical properties such as reduced phonon energy compared with silica glass, transmission from visible (VIS) to midinfrared (MID - IR) approx. $5.5 \mu \mathrm{m}$ possibility of adopting a relatively high concentration of rare earth ions without crystallization or phase separation effect (up to 2 mol\%).

Another group of photonic materials commonly used in photonic applications are polymers (organic glasses) that offer a wide range of advantages, such as: high elasticity, making it possible to produce "flexible" and medical safe optical fibers which are typically characterised by significantly larger diameters (up to several $\mathrm{mm}$ ), high numerical aperture, lower manufacturing costs (extrusion while drawing processes are carried out at a significantly lower temperature compared to glasses, i.e. below $300^{\circ} \mathrm{C}$ ) offering wide-ranging possibilities of doping with functional compounds (organic and inorganic).

In this paper we show our outstanding results on optical fibre technology carried out in tree scientific paths: REdoped germanate optical fiber, plasmonic effect in antimony-germanate glass fibers and RE-dyes coactivated polymeric optical fibres.

Investigations on luminescent properties of the $\mathrm{GeO}_{2}-$ $\mathrm{Ga}_{2} \mathrm{O}_{3}-\mathrm{BaO}$ glass system were related low phonon energy $\left(805 \mathrm{~cm}^{-1}\right)$ resulting in $\mathrm{Ge}-\mathrm{O}(\mathrm{Ge})$ vibrations enabling to obtain emission in a visible and NIR range as a result of donor-acceptor energy transfer and 
superposition of radiative transitions. In order to achieve broadband radiation at the $2 \mu \mathrm{m}$ region, holmium and thulium fluorescence properties are the most promising. The broad emission band, higher stimulated emission cross-section and longer fluorescent lifetime of the upper laser level, make $\mathrm{Tm}^{3+}$ and $\mathrm{Ho}^{3+}$ ions more suitable for generating $2 \mu \mathrm{m}$ laser or broadband ASE radiation. Figure 1 presents the ASE spectrum of a $50 \mathrm{~cm}$ length optical fiber under $796 \mathrm{~nm}$ laser diode excitation. Obtained broadband emission is a result of the superposition of bands related to the $\mathrm{Tm}^{3+}:{ }^{3} \mathrm{H}_{4} \rightarrow{ }^{3} \mathrm{~F}_{4}, \mathrm{Tm}^{3+}:{ }^{3} \mathrm{~F}_{4} \rightarrow{ }^{3} \mathrm{H}_{6}$ and $\mathrm{Ho}^{3+.5} \mathrm{I}_{7} \rightarrow{ }^{5} \mathrm{I}_{8}$ transitions.

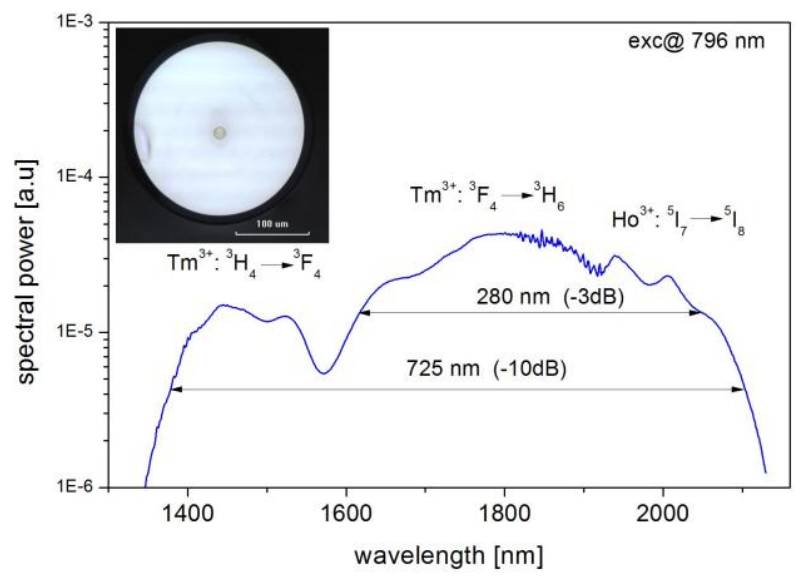

Fig. 1. ASE spectra of double-clad germanate optical fiber co-doped with $\mathrm{Tm}^{3+} / \mathrm{H}^{3+}$, photo of fiber (inset) [2].

The broadband emission (FHWM $=280 \mathrm{~nm}: 3 \mathrm{~dB}$ and FWHM $=725 \mathrm{~nm}: 10 \mathrm{~dB}$ ) is much wider than that presented in silica fiber co-doped with $\mathrm{Tm}^{3+} / \mathrm{Ho}^{3+}$ fabricated by MCVD and solution doping method, where the bandwidth achieved at a level of $-10 \mathrm{~dB}$ was $645 \mathrm{~nm}$. This effect is correlated with our systematic study describing the correlation structure of germanate glasses with their luminescent properties [2]. This is particularly important for optimizing the emission properties (laser parameters) of materials for use in waveguide structures applications (optical fibers) dedicated to work in a specified spectral range. Moreover, the research direction resulting from the method of controlled change of rare earth ions environment in germanate glass allows to obtain higher efficiency of energy transfer between rare earth ions, and thus more efficient luminescence is observed in the visible and infrared spectrum range.

The other research area is the study of Fiber optics codoped with lanthanides and precious metal nanoparticles, concerning the analysis of the plasmon effect for silver and gold nanoparticles placed in the core of optical fiber.
In order to explain the mechanisms responsible for luminescence shaping by interaction of silver nanoparticles with rare earth ions, we conducted research on antimony-germanate glasses to obtain luminescence signal enhancement and possibility of making optical fiber out of them. The confirmation of the plasmon effect is the observation of glass morphology carried out by means of electron microscopy (SEM), where we observed that silver nanoparticles had already formed at the stage of glass synthesis (the inset in Fig. 2). Analyzing luminescence spectra, we found that the transformation of glass into fiber did not bring significant differences in the character of luminescence, which suggests good thermal stability of fabricated glasses. The experiment with annealing glass fiber also brought an unexpected result. Namely, when annealing fibers with a molar content of $0.6 \% \mathrm{AgNO}_{3}$, a thin film with a characteristic amber coloration was formed on their surface. Performing measurements of absorption and observation of fibers under an electron microscope allowed to clearly state that the observed phenomenon is the effect of $\mathrm{Ag}^{+}$ions reduction on the surface of the fiber (Fig. 2). This phenomenon is related to the mechanism of selforganization of silver molecules inside the glass structure under thermal conditions above the transformation temperature.

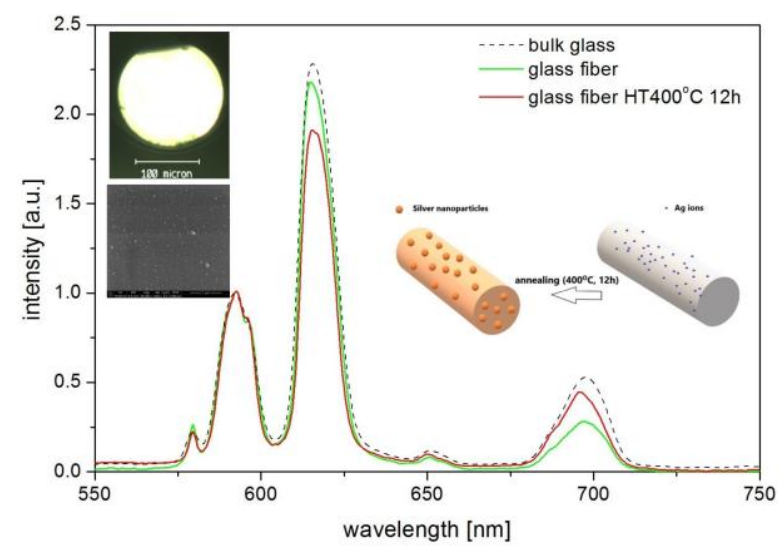

Fig. 2. Luminescence spectra of antimony-germanate fiber glass doped with $\mathrm{Eu}^{3+}$ ions and Ag NPs. Photo of fiber and SEM (inset) [3].

As a result of spectroscopic studies, apart from selfassembly of silver nano-particles in the form of a thin film on the surface of the fiber, there is an increase in the ordering of the structure around the $\mathrm{Eu}^{3+}$ ion (Fig. 2). The results regarding the reduction on the fiber surface and self-assembly of silver nanoparticles in fabricated glasses and optical fibers have a unique character, necessary for functionalization of optical materials used in optical biosensors based on the SERS phenomenon (Surface Enhanced Raman Scattering). 
The third direction of research is focused on functional polymer optical fibers and sensing structures to be applied in the visible spectrum range based on polymeric matrices (e.g. poly(methyl methacrylate (PMMA), Poly(vinyl alcohol) (PVA)). Among the organic compounds, effective luminescence processes in PMMA were observed in xanthenes (Rhodamine B, Rhodamine 6G, Fluorescein) and aromatic hydrocarbons (Perylene, 1,4Bis(2-methylstyryl) benzene (Bis-MSB)) [4]. Typically, they have broad excitation and emission bands resulting from partial disappearance of oscillations of structural groups occurring as a result of mutual interactions between chromophores and the surrounding medium. Most organic dyes are also characterized by a short luminescence time that lasts nanoseconds. On the other hand, lanthanides (well known from silica and soft glass fibers lasers and amplifiers technology) with a long luminescence decay time and a well-defined emission spectrum profile are interesting for new optical applications. In such circumstances, the polymer matrix can be effectively doped by organometallic complexes of lanthanides. The carried research showed that (Terbium (III) -tris- (2,2,6,6-tetramethyl-3,5-heptanedionate), Europium(III) -tris- (2,2,6,6-tetramethyl-3,5heptanedionate), Dysprosium(III) -tris- $\quad(2,2,6,6$ tetramethyl-3,5-heptanedionate) can be successfully applied for poly(methyl methacrylate) doping. The advantage of using organometallic complexes is a wide excitation band thanks to energy transfer from organic ligand to luminescent rare earth ion (antenna effect). It is well known that a co-doping process of luminescent dyes/lanthanides can be used for energy transfer investigations (e.g. Perylene-Rhodamine $6 \mathrm{G}, \mathrm{Tb}^{3+}-\mathrm{Eu}^{3+}$, $\mathrm{Tb}^{3+}$-Rhodamine B) and new luminescent properties of optical fibers constructions. A combination of dopants can be used for some specific luminescence profile in polymeric optical fibers (Fig. 3). Wide emission of Perylene-Rhodamine 6G (420-620 nm) was used for a white light fluorescent fiber illuminator. Moreover, new photochromic features of poly(methyl methacrylate can be obtained by embedding the Spirooxazine (absorption band in the range from $\lambda=460 \mathrm{~nm}$ to $680 \mathrm{~nm}$ with a maximum wavelength of $\lambda=602 \mathrm{~nm}$ for exposition to a mercury vapor lamp, at a wavelength of $\lambda=365 \mathrm{~nm}, 21.5 \mathrm{~W} / \mathrm{m}^{2}$ ) and 1,4-Bis(2-methylstyryl) benzene (wide absorption band below a wavelength of $\lambda=670 \mathrm{~nm}$ with four characteristic absorption bands at $\lambda=435,516,565$, $626 \mathrm{~nm}$ for exposition to Nd-YAG laser, third harmonic $\lambda=355 \mathrm{~nm}$ wavelength, $4 \mathrm{~mJ}$ energy, pulse width $3-5 \mathrm{~ns}$ ).

http://www.photonics.pl/PLP

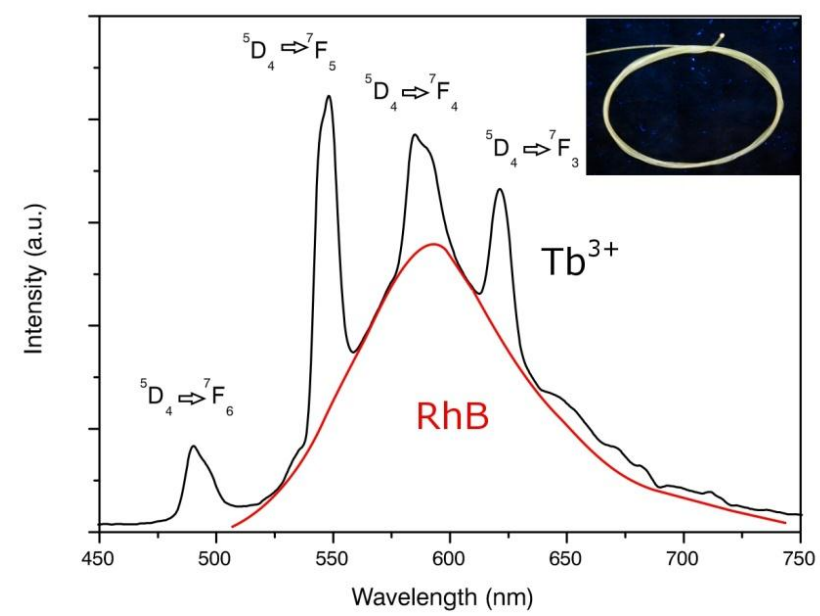

Fig. 3. Luminescence spectrum of $\mathrm{Tb}^{3+}$ Rhodamine B co-doped PMMA fibre (diameter $0.9 \mathrm{~mm}$, length $40 \mathrm{~mm}$, at exc. $355 \mathrm{~nm}$ ), inset: fiber under $365 \mathrm{~nm}$ (mercury vapor black light) excitation

The changing of optical transmission is possible due to molecule structure transformation under high energy UV radiation. All of these materials were successfully used for fabrication of cylindrical fiber structures, which additionally allows for significant modification of the emission spectrum profile by using: the reabsorption and reemission process, spectral attenuation, energy transfer between luminescent compounds and local modifications of molecules structures. The incorporation process and structural transformations of different functional complexes in a polymer matrix are crucial for developing new functional polymers.

In conclusions, the presented results are related to original scientific achievements and constitute a significant contribution to the field of knowledge regarding the luminescent properties of optical glasses and polymers in the visible and near infrared range, for the needs of modern optical fiber photonics.

All presented results were funded by the National Science Centre (Poland) granted on the basis of the decisions No. DEC2016/21/D/ST7/03453; DEC-2016/23/B/ST8/00706; DEC2017/01/X/ST8/00595.

\section{References}

[1] A. Zajac, D. Dorosz, M. Kochanowicz, M. Skórczakowski, J. Świderski, Bull. Pol. Ac.: Tech. 58, 4 (2010).

[2] M. Kochanowicz, J. Zmojda, P. Miluski, A. Baranowska, M. Leich, A. Schwuchow, M. Jager, M. Kuwik, J. Pisarska, W.A. Pisarski, D. Dorosz, Opt. Mat. Expr. 9, 3 (2019).

[3] J. Zmojda, M. Kochanowicz, P. Miluski, W.A., Pisarski, J. Pisarska, R. Jadach, M. Sitarz, D. Dorosz, Spectrochimica Acta - Part A: Molecular and Biomolecular Spectroscopy 201 (2018).

[4] P. Miluski, M. Kochanowicz, J. Zmojda, A. Baranowska, D. Dorosz, Opt. Materials 87, 132 (2019). 